\title{
The checklist of Latvian spiders (Arachnida: Araneae)
}

\author{
Inese Cera* \\ Laboratory of Bioindication, Institute of Biology, University of Latvia, Miera 3, Salaspils LV-2169, Latvia \\ *Corresponding author, E-mail: inese.cera@gmail.com
}

\begin{abstract}
The checklist includes 500 spider species of 31 families mentioned in 74 publications and manuscripts, covering all previous studies on spiders in the territory of Latvia known to the author.
\end{abstract}

Key words: Araneae, Latvia, spider species.

\section{Introduction}

Spiders are distributed worldwide and now the known number of species is more than 47520 (World Spider Catalog 2018). They can be found in different habitats, even under water (Foelix 1996).

Studies on spiders in Latvia were started in the $19^{\text {th }}$ century by A.E. Grube (1859). After his work in the middle of the $20^{\text {th }}$ century M. Sternbergs studied spiders in different habitats (beginning in 1974, the last publication was in 1998). After the research of Šternebrgs no more intensive spider studies were conducted until the beggining of the $21^{\text {st }}$ century, starting when V. Spungis (2005) began new studies.

The last chek list of spider species of Latvia was published in 2002 (Relys, Spungis 2002) and it is incomplete. Several new studies on spiders were conducted, and new species for Latvia were identified. This publication suplemments the list of spider species with more than 30 new entries as well as corrects previous mistakes or misunderstandings, thus giving more clear information on the current situation in arachnology in Latvia.

\section{Methods}

This checklist analyses 74 publications and manuscripts on fauna of Latvian spiders beginning with the A.E. Grube (1859) in the $19^{\text {th }}$ century, followed by M. Šternbergs (1974; 1976; 1977; 1979a; 1979b; 1980a; 1980b; 1980c; 1981a; 1981b; 1982; 1982b; 1984; 1985a; 1985b; 1986; 1987; 1988; 1990a; 1991; 1995a; 1995b; 1995c; 1998; 1989) and with co-authors (Prieditis, Stenbergs 1981; 1882; Ancipanova, Stenbergs 1984; 1987; Karps et al. 1990b), and diploma thesis of his students (Fedorova 1991; Sevcenko 1991; Vārtiṇa 1992; Brūvele 1993; Smal̦inskis 1994; Kušķ̣e 1995; Petrovics 1995; Tkaceva 1996; Samulaka 1996; Golubeva 1997; Narodickaja 1997). Šternbergs also identified spiders in other studies related to invertebrate fauna on agriculture land; these results are not published but they are summarized in several reports (Mihnevics et al. 1989; 1990). The most recent research has been carried out by the author of this article (Cera 2008; 2009; 2013) and with coauthors (Cera, Spunggis 2008; 2010; Cera et al. 2010; Cera, Spungóis 2013; Petrova et al. 2013; Cera, Keišs 2016), and also by M. Štokmane alone (2013) and with co-authors (Štokmane et al. 2013; Štokmane, Spungis 2014).

Šternbergs published the first check list of Latvian spiders in the journal Latvijas Entomologs (initially beginning with the Salticidae; Strenbergs 1974), the last part of his checklist was published in 1998 (Šternbergs 1998). Relys and Spuñgis published the checklist of Latvian spiders on-line in 2002 (Relys, Spunggis 2002). Complex investigations of Latvia spider fauna were carried out during the last decade (by the author of this article and Štokmane et al. 2013; also Spungis et al. 2005 and his student (Šulmeistare 1998). These investigations covered various habitats: dunes, peat bogs, coastal meadows and calcareous fens of coastal lowlands.

The species Argipe bruennichi (Araneidae) was described as new species for Latvia by Spungis (2005). Another new species, Gibbaranea omoeda (Araneidae), was also discovered in historical collections of spiders in the Museum für Naturkunde der Humboldt Universität zu Berlin, Germany (Šestáková, Krumpál 2013). Aditionally, five species (Marpissa muscosa, Neon levis, Neon pictus, Segestria senoculata and Talavera aequipes 2015) were included in the checklist after the revision (done by the author of this article) of the spider collection of the Latvian Museum of Natural History (collected by M. Šternbergs); these species have not been included in any publication before the present one.

The following spider species were included in the species list of Latvia by Relys and Spungigis (2002), but have no published references or collected specimens available, and therefore they are not included in this checklist: Aphilleta 
Table 1. List of spider families, genera and species of Latvia

\begin{tabular}{|c|c|c|}
\hline Family & Genus & Species \\
\hline Pholicidae & Pholcus Walckenaer, 1805 & Pholcus phalangoides (Fuesslin, 1775) \\
\hline Segestriidae & Segestria Latreille, 1804 & Segestria senocula (Linnaeus, 1758) \\
\hline Dysderidae & Harpactea Bristowe, 1939 & Harpactea rubicunda (C. L. Koch, 1838) \\
\hline \multirow[t]{2}{*}{ Mimetidae } & Ero C. L. Koch, 1836 & Ero cambridgei Kulczyński, 1911 \\
\hline & & Ero furcata (Villers, 1789) \\
\hline Eresidae & Eresus Walckenaer, 1805 & Eresus sandaliatus (Martini \& Goeze, 1778) \\
\hline Oecobiidae & Oecobius Lucas, 1846 & Oecobius navus Blachwall, 1859 \\
\hline Uloboridae & Hyptiotes Walckenaer, 1837 & Hyptiotes paradoxus (C. L. Koch, 1834) \\
\hline Nesticidae & Nesticus Thorell, 1869 & Nesticus cellulanus (Clerck, 1757) \\
\hline \multirow[t]{37}{*}{ Theridiidae } & Anelosimus Simon, 1891 & Anelosimus pulchellus (Walckenaer, 1802) \\
\hline & Asagena Sundevall, 1833 & Asagena phalerata (Panzer, 1801) \\
\hline & Cryptachaea Archer, 1946 & Cryptachaea riparia (Blackwall, 1834) \\
\hline & Crustulina Menge, 1868 & Crustulina guttata (Wider, 1834) \\
\hline & & Crustulina sticta (O. Pickard-Cambridge, 1861) \\
\hline & Enoplognatha Pavesi, 1880 & Enoplognatha ovata (Clerck, 1757) \\
\hline & & Enoplognatha thoracica (Hahn, 1833) \\
\hline & Episinus Walckenaer, in Latreille 1809 & Episinus angulatus (Blackwall, 1836) \\
\hline & & Episinus truncatus Latreille, 1809 \\
\hline & Euryopis Menge, 1868 & Euryopis flavomaculata (C. L. Koch, 1836) \\
\hline & Lasaeola Simon, 1881 & Lasaeola prona (Menge, 1868) \\
\hline & & Lasaeola tristis (Hahn, 1833) \\
\hline & Neottiura Menge, 1869 & Neottiura bimaculata (Linnaeus, 1767) \\
\hline & Paidiscura Archer, 1950 & Paidiscura pallens (Blackwall, 1834) \\
\hline & Parasteatoda Archer, 1946 & Parasteatoda lunata (Clerck, 1757) \\
\hline & & Parasteatoda simulants (Thorell, 1875) \\
\hline & & Parasteatoda tepidariorum (C. L. Koch, 1841) \\
\hline & Pholcomma Thorell, 1869 & Pholcomma gibbum (Westring, 1851) \\
\hline & Phylloneta Archer, 1950 & Phylloneta impressa (L. Koch, 1881) \\
\hline & & Phylloneta sisyphia (Clerck, 1757) \\
\hline & Platnickina Koçak, Kemal, 2008 & Platnickina tincta (Walckenaer, 1802) \\
\hline & Robertus O. P.-Cambridge, 1879 & Robertus arundineti (O. P.-Cambridge, 1871) \\
\hline & & Robertus insignis O. P.-Cambridge, 1907 \\
\hline & & Robertus neglectus (O. P.-Cambridge, 1871) \\
\hline & & Robertus lividus (Blackwall, 1836) \\
\hline & & Robertus scoticus Jackson, 1914 \\
\hline & Simitidion Wunderlich, 1992 & Simitidion simile (C. L. Koch, 1836) \\
\hline & Steatoda Sundevall, 1833 & Steatoda albomaculata (De Geer, 1778) \\
\hline & & Steatoda bipunctata (Linnaeus, 1758) \\
\hline & & Steatoda castanea (Clerck, 1757) \\
\hline & & Steatoda grossa (C. L. Koch, 1838) \\
\hline & & Steatoda triangulosa (Walckenaer, 1802) \\
\hline & Theonoe Simon, 1881 & Theonoe minutissima (O. P.-Cambridge, 1879) \\
\hline & Theridion Walckenaer, 1805 & Theridion familiare O. P.-Cambridge, 1871 \\
\hline & & Theridion melanurum Hahn, 1831 \\
\hline & & Theridion pictum (Walckenaer, 1802) \\
\hline & & Theridion variants Hahn, 1833 \\
\hline \multirow[t]{4}{*}{ Linyphiidae } & Abacoproeces Simon, 1844 & Abacoproeces saltuum (L. Koch, 1872) \\
\hline & Agyneta Hull, 1911 & Agyneta affinis (Kulczyński, 1898) \\
\hline & & Agyneta cauta (O. Pickard-Cambridge, 1902) \\
\hline & & Agyneta conigera (O. Pickard-Cambridge, 1863) \\
\hline
\end{tabular}


Table 1. continued

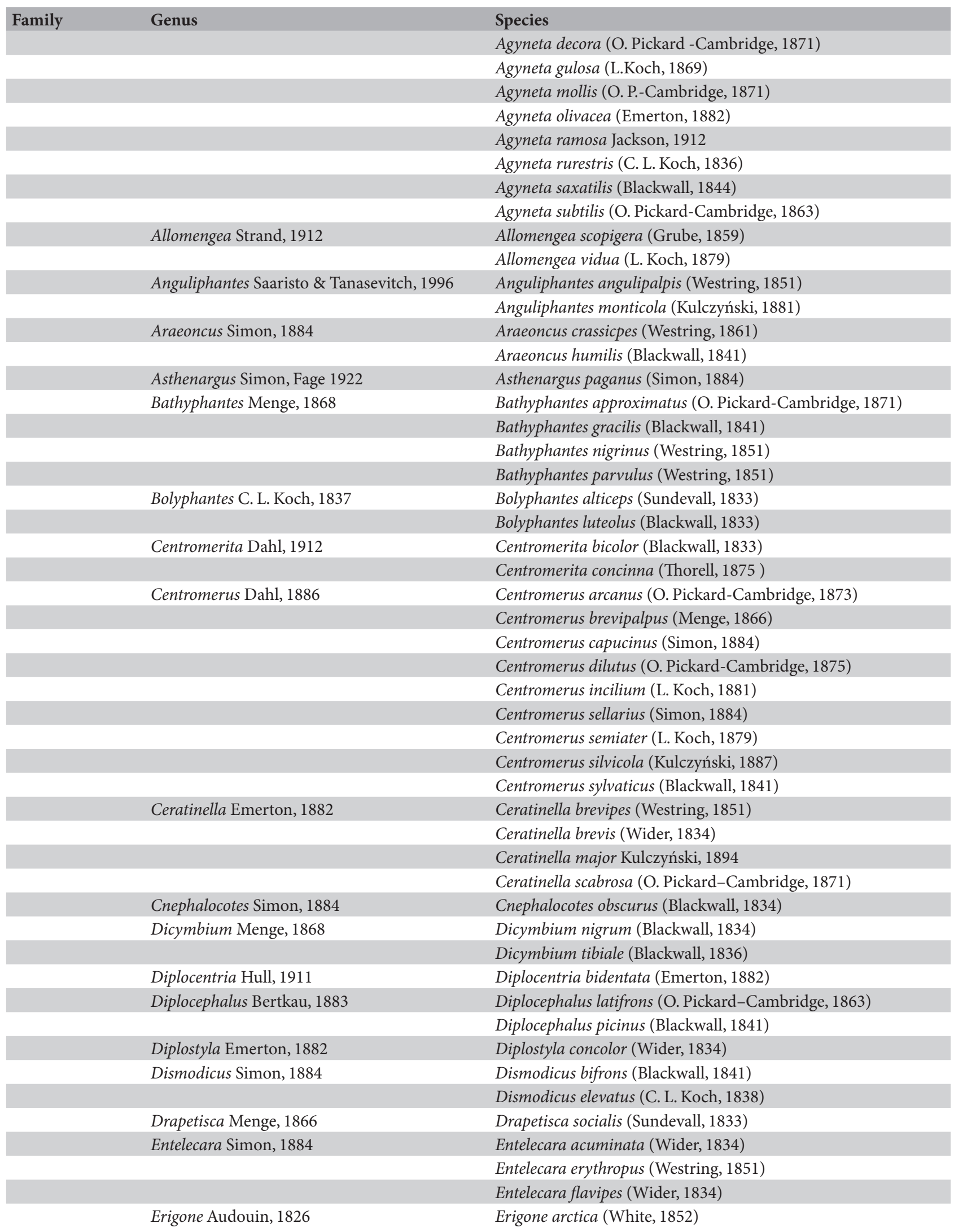

continued 
Table 1. continued

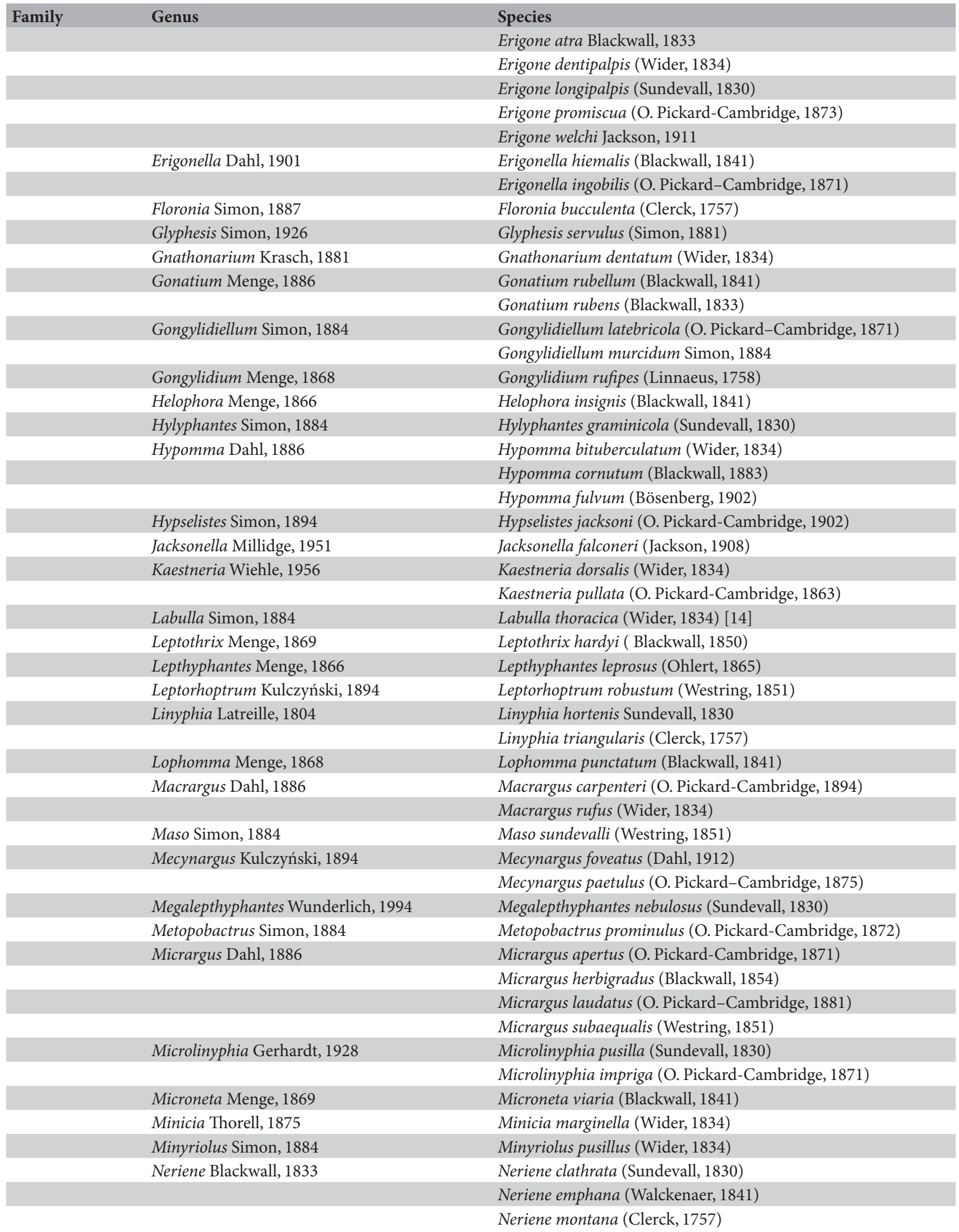


Table 1. continued

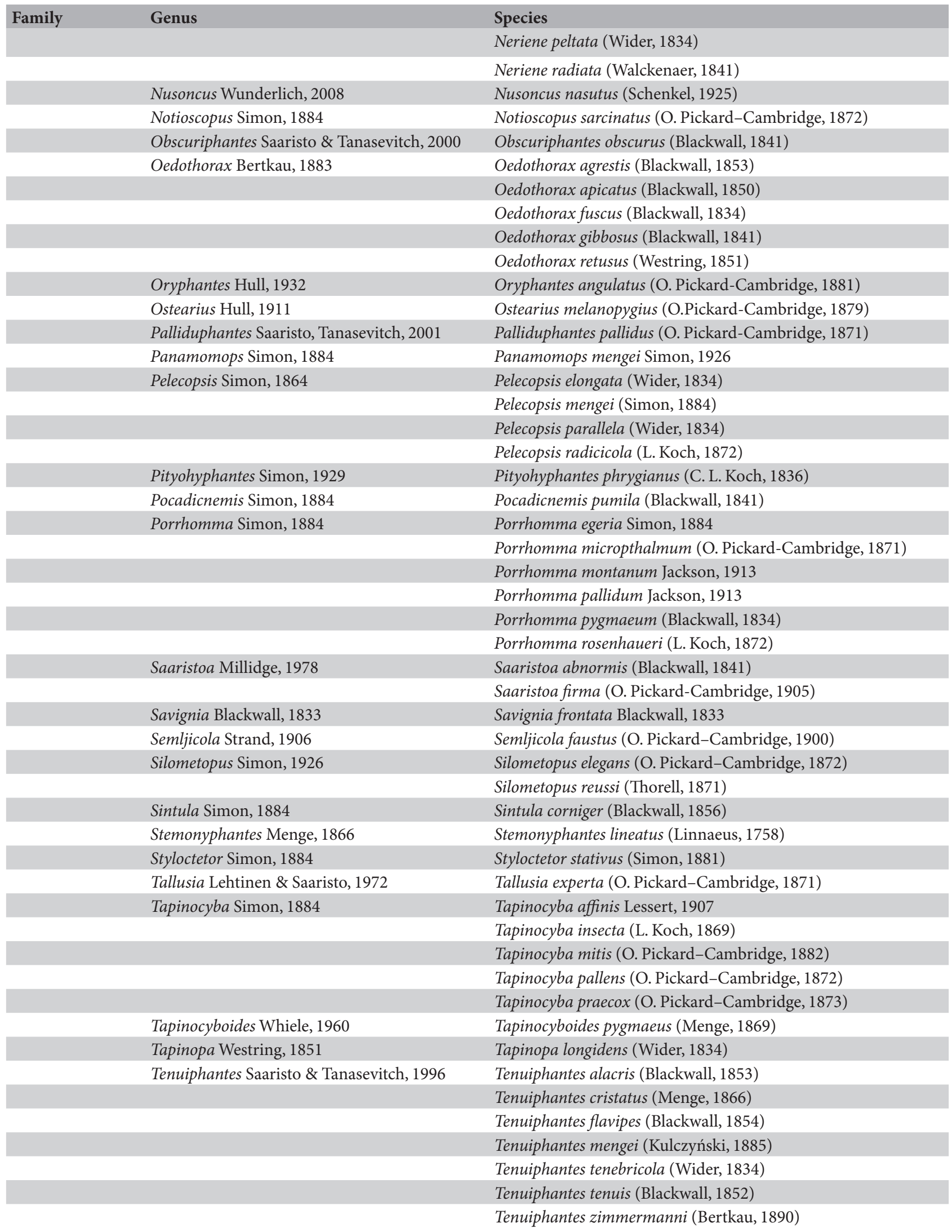

continued 
Table 1. continued

\begin{tabular}{|c|c|c|}
\hline \multirow[t]{27}{*}{ Family } & Genus & Species \\
\hline & Thyreosthenius Simon, 1884 & Thyreosthenius biovatus (O. Pickard-Cambridge, 1875) \\
\hline & & Thyreosthenius parasiticus (Westring, 1851) \\
\hline & Tiso Simon, 1884 & Tiso vagans (Blackwall, 1834) \\
\hline & Tmeticus Menge, 1868 & Tmeticus affinis (Blackwall, 1855) \\
\hline & Trematocephalus Dahl, 1886 & Trematocephalus cristatus (Wider, 1834) \\
\hline & Trichopterna Kulczyński, 1894 & Trichopterna cito (O. Pickard-Cambridge, 1872) \\
\hline & Trichopternoides Wunderlich, 2008 & Trichopternoides thorelli (Westring, 1861) \\
\hline & Troxochrus Simon, 1884 & Troxochrus scabriculus (Westring, 1851) \\
\hline & Typhochrestus Simon, 1884 & Typhochrestus digitatus (O. Pickard-Cambridge, 1872) \\
\hline & Walckenaeria Blackwall, 1833 & Walckenaeria acuminata Blackwall, 1833 \\
\hline & & Walckenaeria alticeps (Denis, 1952) \\
\hline & & Walckenaeria antica (Wider, 1834) \\
\hline & & Walckenaeria atrotibialis (O. Pickard-Cambridge, 1878) \\
\hline & & Walckenaeria capito (Westring, 1861) \\
\hline & & Walckenaeria corniculans (O. Pickard-Cambridge, 1875) \\
\hline & & Walckenaeria cucullata (C. L. Koch, 1836) \\
\hline & & Walckenaeria cuspidata Blackwall, 1833 \\
\hline & & Walckenaeria dysderoides (Wider, 1834) \\
\hline & & Walckenaeria furcillata (Menge, 1869) \\
\hline & & Walckenaeria kochi (O. Pickard-Cambridge, 1872) \\
\hline & & Walckenaeria mitrata (Menge, 1868) \\
\hline & & Walckenaeria nodosa O. Pickard-Cambridge, 1873 \\
\hline & & Walckenaeria nudipalpis (Westring, 1851) \\
\hline & & Walckenaeria obtusa Blackwall, 1836 \\
\hline & & Walckenaeria unicornis O. Pickard-Cambridge, 1861 \\
\hline & & Walckenaeria vigilax (Blackwall, 1853) \\
\hline \multirow[t]{13}{*}{ Tetragnathidae } & Meta C. L. Koch, 1836 & Meta menardi (Latreille, 1804) \\
\hline & Metellina Chamberlin, Ivie 1941 & Metellina mengei (Blackwall, 1869) \\
\hline & & Metellina merianae (Scopoli, 1763) \\
\hline & & Metellina segmentata (Clerck, 1757) \\
\hline & Pachygnatha Sundevall, 1823 & Pachygnatha clercki Sundevall, 1823 \\
\hline & & Pachygnatha degeeri Sundevall, 1830 \\
\hline & & Pachygnatha listeri Sundevall, 1830 \\
\hline & Tetragnatha Latreille, 1804 & Tetragnatha dearmata Thorell, 1873 \\
\hline & & Tetragnatha extensa (Linnaeus, 1758) \\
\hline & & Tetragnatha montana Simon, 1874 \\
\hline & & Tetragnatha nigrita Lendl, 1886 \\
\hline & & Tetragnatha obtusa C. L. Koch, 1837 \\
\hline & & Tetragnatha pinicola L. Koch, 1870 \\
\hline \multirow[t]{11}{*}{ Araneidae } & Aculepeira Chamberlin, Ivie 1942 & Aculepeira ceropegia (Walckenaer, 1802) \\
\hline & Agalenatea Archer, 1951 & Agalenatea redii (Scopili, 1763) \\
\hline & Araneus Clerck, 1757 & Araneus alsine (Walckenaer, 1802) \\
\hline & & Araneus angulatus Clerck, 1757 \\
\hline & & Araneus diadematus Clerck, 1757 \\
\hline & & Araneus marmoreus Clerck, 1757 \\
\hline & & Araneus quadratus Clerck, 1757 \\
\hline & & Araneus saevus (L. Koch, 1872) \\
\hline & & Araneus sturmi (Hahn, 1831) \\
\hline & & Araneus triguttatus (Fabricius, 1793) \\
\hline & Araniella Chamberlin, Ivie 1942 & Araniella alpica (L. Koch, 1869) \\
\hline
\end{tabular}

continued 
Table 1. continued

\begin{tabular}{|c|c|c|}
\hline \multirow[t]{26}{*}{ Family } & Genus & Species \\
\hline & & Araniella cucurbitina (Clerck, 1757) \\
\hline & & Araniella inconspicua (Simon, 1874) \\
\hline & & Araniella proxima (Kulczyński, 1885) \\
\hline & Argiope Audouin, 1826 & Argiope bruennichi (Scopoli, 1772) \\
\hline & Cercidia Thorell, 1869 & Cercidia prominens (Westring, 1851) \\
\hline & Cyclosa Menge, 1866 & Cyclosa conica (Pallas, 1772) \\
\hline & & Cyclosa oculata (Walckenaer, 1802) \\
\hline & Gibbaranea Archer, 1951 & Gibbaranea bituberculata (Walckenaer, 1802) \\
\hline & & Gibbaranea omoeda (Thorell, 1870) \\
\hline & Hypsosinga Ausserer, 1871 & Hypsosinga heri (Hahn, 1831) \\
\hline & & Hypsosinga pygmaea (Sundevall, 1831) \\
\hline & & Hypsosinga sanguinea (C. L. Koch, 1844) \\
\hline & Larinioides Caporiacco, 1934 & Larinioides cornutus (Clerck, 1757) \\
\hline & & Larinioides patagiatus (Clerck, 1757) \\
\hline & & Larinioides sericatus (Clerck, 1757) \\
\hline & Mangora O. Pickard-Cambridge, 1889 & Mangora acalypha (Walckenaer, 1802) \\
\hline & Neocsona Simon, 1864 & Neocsona adianta (Walckenaer, 1802) \\
\hline & Nuctenea Simon, 1864 & Nuctenea silvicultrix (C. L. Koch, 1835) \\
\hline & & Nuctenea umbratica (Clerck, 1757) \\
\hline & Singa C. L. Koch, 1836 & Singa hamata (Clerck, 1757) \\
\hline & & Singa nitidula C. L. Koch, 1844 \\
\hline & Stroemiellus Wunderlich, 2004 & Stroemiellus stroemi (Thorell, 1870) \\
\hline & Zilla C. L. Koch, 1834 & Zilla diodia (Walckenaer, 1802) \\
\hline & Zygiella F. O. Pickard-Cambridge, 1902 & Zygiella atrica (C. L. Koch, 1845) \\
\hline & & Zygiella $x$-notata (Clerck, 1757) \\
\hline \multirow[t]{25}{*}{ Lycosidae } & Acantholycosa Dahl, 1908 & Acantholycosa lignaria (Clerck, 1757) \\
\hline & Alopecosa Simon, 1885 & Alopecosa aculeata (Clerck, 1757) \\
\hline & & Alopecosa cuneata (Clerck, 1757) \\
\hline & & Alopecosa cursor (Hahn, 1831) \\
\hline & & Alopecosa fabrilis (Clerck, 1757) \\
\hline & & Alopecosa inquilina (Clerck, 1757) \\
\hline & & Alopecosa pulverulenta (Clerck, 1757) \\
\hline & & Alopecosa schmidti (Hahn, 1835) \\
\hline & & Alopecosa trabalis ( Clerck, 1757) \\
\hline & Arctosa C. L. Koch, 1847 & Arctosa alpigena (Doleschall, 1852) \\
\hline & & Arctosa cinerea (Fabricius, 1777) \\
\hline & & Arctosa figurata (Simon, 1876) \\
\hline & & Arctosa leopardus (Sundevall, 1833) \\
\hline & & Arctosa lutetiana (Simon, 1876) \\
\hline & & Arctosa maculata (Hahn, 1822) \\
\hline & & Arctosa perita (Latreille, 1799) \\
\hline & & Arctosa stigmosa (Thorell, 1875) \\
\hline & Aulonia C. L. Koch, 1847 & Aulonia albimana (Walckenaer, 1805) \\
\hline & Hygrolycosa Dahl, 1908 & Hygrolycosa rubrofasciata (Ohlert, 1865) \\
\hline & Pardosa C. L. Koch, 1847 & Pardosa agrestis (Westring, 1861) \\
\hline & & Pardosa agricola (Thorell, 1856) \\
\hline & & Pardosa amentata (Clerck, 1757) \\
\hline & & Pardosa atrata (Thorell, 1873) \\
\hline & & Pardosa fulvipes (Collett, 1876) \\
\hline & & Pardosa hortensis (Thorell, 1872) \\
\hline
\end{tabular}

continued 
Table 1. continued

\begin{tabular}{|c|c|c|}
\hline \multirow[t]{29}{*}{ Family } & Genus & Species \\
\hline & & Pardosa hyperborea (Thorell, 1872) \\
\hline & & Pardosa lugubris (Walckenaer, 1802) \\
\hline & & Pardosa monticola (Clerck, 1757) \\
\hline & & Pardosa nigriceps (Thorell, 1856) \\
\hline & & Pardosa pauludicola (Clerck, 1757) \\
\hline & & Pardosa palustris (Linnaeus, 1758) \\
\hline & & Pardosa prativaga (L. Koch, 1870) \\
\hline & & Pardosa proxima (C. L. Koch, 1847) \\
\hline & & Pardosa pullata (Clerck, 1757) \\
\hline & & Pardosa riparia (C. L. Koch, 1833) \\
\hline & & Pardosa saltans Töpfer-Hofmann, 2000 \\
\hline & & Pardosa saltuaria (L. Koch, 1870) \\
\hline & & Pardosa sphagnicola (Dahl, 1908) \\
\hline & & Pardosa wagleri (Hahn, 1822) \\
\hline & Pirata Sundevall, 1833 & Pirata piraticus (Clerck, 1757) \\
\hline & & Pirata piscatorius (Clerck, 1757) \\
\hline & & Pirata tenuitarsis Simon, 1876 \\
\hline & & Pirata uliginosus (Thorell, 1856) \\
\hline & Piratula Roewer, 1960 & Piratula hygrophila (Thorell, 1872) \\
\hline & & Piratula insularis (Emerton, 1885) \\
\hline & & Piratula knorri (Scopoli, 1763) \\
\hline & & Piratula latitans (Blackwall, 1841) \\
\hline & Trochosa C. L. Koch, 1847 & Trochosa robusta (Simon, 1876) \\
\hline & & Trochosa ruricola (De Geer, 1778) \\
\hline & & Trochosa spinipalpis (F. O. Pickard-Cambridge, 1895) \\
\hline & & Trochosa terricola Thorell, 1856 \\
\hline & Xerolycosa Dahl, 1908 & Xerolycosa minata (C. L. Koch, 1834) \\
\hline & & Xerolycosa nemoralis (Westring, 1861) \\
\hline \multirow[t]{3}{*}{ Pisauridae } & Dolomedes Latreille, 1804 & Dolomedes fimbriatus (Clerck, 1757) \\
\hline & & Dolomedes plantarius (Clerck, 1757) \\
\hline & Pisaura Simon, 1885 & Pisaura mirabilis (Clerck, 1757) \\
\hline Oxyopidae & Oxyopes Latreille, 1804 & Oxyopes ramosus (Martini, Goeze, 1778) \\
\hline \multirow[t]{3}{*}{ Agelenidae } & Agelena Walckenaer, 1805 & Agelena labyrinthica (Clerck, 1757) \\
\hline & Eratigena Bolzern, Burckhardt, Hänggi, 2013 & Eratigena atrica (C. L. Koch, 1843) \\
\hline & Tegenaria Latreille, 1804 & Tegenaria domestica (Clerck, 1757) \\
\hline Cybaeidae & Argyroneta Latreille, 1804 & Argyroneta aquatica (Clerck, 1757) \\
\hline \multirow[t]{6}{*}{ Hahniidae } & Antistea Simon, 1898 & Antistea elegans (Blackwall, 1841) \\
\hline & Cryphoeca Thorell, 1870 & Cryphoeca silvicola (C. L. Koch, 1834) \\
\hline & Hahnia C. L. Koch, 1841 & Hahnia nava (Blackwall, 1841) \\
\hline & & Hahnia ononidum Simon, 1875 \\
\hline & & Hahnia pusilla C. L. Koch, 1841 \\
\hline & Iberina Simon, 1881 & Iberina montana (Blackwall, 1841) \\
\hline \multirow[t]{5}{*}{ Dictynidae } & Argenna Thorell, 1870 & Argenna subnigra (O. Pickard-Cambridge, 1861) \\
\hline & Dictyna Sundevall, 1833 & Dictyna arundinacea (Linnaeus, 1758) \\
\hline & & Dictyna pusilla Thorell, 1856 \\
\hline & & Dictyna uncinata Thorell, 1856 \\
\hline & Lathys Simon, 1884 & Lathys humilis (Blackwall, 1855) \\
\hline Amaurobiidae & Amaurobius C. L. Koch, 1837 & Amaurobius fenestralis (Ström, 1768) \\
\hline \multirow[t]{2}{*}{ Eutichuridae } & Cheiracanthium C. L. Koch, 1839 & Cheiracanthium elegans Thorell, 1875 \\
\hline & & Cheiracanthium erraticum (Walckenaer, 1802) \\
\hline
\end{tabular}

continued 
Table 1. continued

\begin{tabular}{|c|c|c|}
\hline \multirow[t]{4}{*}{ Family } & Genus & Species \\
\hline & & Cheiracanthium oncognathum Thorell, 1871 \\
\hline & & Cheiracanthium punctorium (Villers, 1789) \\
\hline & & Cheiracanthium virescens (Sundevall, 1833) \\
\hline \multirow[t]{4}{*}{ Miturgidae } & Zora C. L. Koch, 1847 & Zora armillata Simon, 1878 \\
\hline & & Zora nemoralis (Blackwall, 1861) \\
\hline & & Zora silvestris Kulczyński, 1897 \\
\hline & & Zora spinimana (Sundevall, 1833) \\
\hline Anyphaenidae & Anyphaena Sundevall, 1833 & Anyphaena accentuata (Walckenaer, 1802) \\
\hline \multirow[t]{7}{*}{ Liocranidae } & Agroeca Westring, 1861 & Agroeca brunnea (Blackwall, 1833) \\
\hline & & Agroeca dentigera Kulczyński, 1913 \\
\hline & & Agroeca lusatica (L. Koch, 1875) \\
\hline & & Agroeca proxima (O. Pickard-Cambridge, 1871) \\
\hline & Liocranoeca Wunderlich, 1999 & Liocranoeca striata (Kulczyński, 1882) \\
\hline & Scotina Menge, 1873 & Scotina gracilipes (Blackwall, 1859) \\
\hline & & Scotina palliardii (L Koch, 1881) \\
\hline Phrurolithidae & Phrurolithus C. L. Koch, 1839 & Phrurolithus festivus (C. L. Koch, 1835) \\
\hline \multirow[t]{17}{*}{ Clubionidae } & Clubiona Latreille, 1804 & Clubiona caerulescens L. Koch, 1867 \\
\hline & & Clubiona comta C. L. Koch, 1839 \\
\hline & & Clubiona decora Blackwall, 1859 \\
\hline & & Clubiona diversa O. Pickard-Cambridge, 1862 \\
\hline & & Clubiona frisia Wunderlich, Schuett, 1995 \\
\hline & & Clubiona frutetorum L Koch, 1867 \\
\hline & & Clubiona germanica Thorell, 1871 \\
\hline & & Clubiona lutescens Westring, 1851 \\
\hline & & Clubiona neglecta O. Pickard-Cambridge, 1862 \\
\hline & & Clubiona pallidula (Clerck, 1757) \\
\hline & & Clubiona phragmitis C. L. Koch, 1843 \\
\hline & & Clubiona reclusa O. Pickard-Cambridge, 1863 \\
\hline & & Clubiona similis L. Koch, 1867 \\
\hline & & Clubiona stagnatilis Kulczyński, 1897 \\
\hline & & Clubiona subsultans Thorell, 1875 \\
\hline & & Clubiona subtilis L. Koch, 1867 \\
\hline & & Clubiona trivialis C. L. Koch, 1843 \\
\hline \multirow[t]{17}{*}{ Gnaphosidae } & Callilepis Westring, 1874 & Callilepis nocturna (Linnaeus, 1758) \\
\hline & Drassodes Westring, 1851 & Drassodes lapidosus (Walckenaer, 1802) \\
\hline & & Drassodes pubescens (Thorell, 1856) \\
\hline & Drassyllus Chamberlin, 1922 & Drassyllus lutetianus (L. Koch, 1866) \\
\hline & & Drassyllus praeficus (L. Koch, 1866) \\
\hline & & Drassyllus pusillus (C. L. Koch, 1833) \\
\hline & Gnaphosa Latreille, 1804 & Gnaphosa bicolor (Hahn,1833) \\
\hline & & Gnaphosa lapponum (L. Koch, 1866) \\
\hline & & Gnaphosa leporina (L. Koch, 1866) \\
\hline & & Gnaphosa lucifuga (Walckenaer, 1802) \\
\hline & & Gnaphosa lugubris (C. L. Koch, 1839) \\
\hline & & Gnaphosa montana (L. Koch, 1866) \\
\hline & & Gnaphosa muscorum (L. Koch, 1866) \\
\hline & & Gnaphosa nigerrima L. Koch, 1877 \\
\hline & Haplodrassus Chamberlin, 1922 & Haplodrassus cognatus (Westring, 1861) \\
\hline & & Haplodrassus moderatus (Kulczyński, 1897) \\
\hline & & Haplodrassus signifer (C. L. Koch, 1839) \\
\hline
\end{tabular}

continued 
Table 1. continued

\begin{tabular}{|c|c|c|}
\hline \multirow[t]{22}{*}{ Family } & Genus & Species \\
\hline & & Haplodrassus silvestris (Blackwall, 1833) \\
\hline & & Haplodrassus soerenseni (Strand, 1900) \\
\hline & & Haplodrassus umbratilis (L. Koch, 1866) \\
\hline & Micaria Westring, 1851 & Micaria albovittata (Lucas, 1846) \\
\hline & & Micaria fulgens (Walckenaer, 1802) \\
\hline & & Micaria lenzi Bösenberg, 1899 \\
\hline & & Micaria pulicaria (Sundevall, 1831) \\
\hline & & Micaria silesiaca L. Koch, 1875 \\
\hline & & Micaria subopaca Westring, 1861 \\
\hline & Phaeocedus Simon, 1893 & Phaeocedus braccatus (L. Koch, 1866) \\
\hline & Poecilochora Westring, 1874 & Poecilochora variana ( C. L. Koch, 1839) \\
\hline & Scotophaeus Simon, 1893 & Scotophaeus quadripunctatus (Linnaeus, 1758) \\
\hline & Sosticus Chamberlin, 1922 & Sosticus loricatus (L. Koch, 1866) \\
\hline & Trachyzelotes Lohmander, 1944 & Trachyzelotes pedestris (C. L. Koch, 1837) \\
\hline & Zelotes Gistel, 1848 & Zelotes apricorum (L. Koch, 1876) \\
\hline & & Zelotes clivicola (L. Koch, 1870) \\
\hline & & Zelotes electus (C. L. Koch, 1839) \\
\hline & & Zelotes latreillei (Simon, 1878) \\
\hline & & Zelotes longipes (L. Koch, 1866) \\
\hline & & Zelotes petrensis (C. L. Koch, 1839) \\
\hline & & Zelotes subterraneus (C. L. Koch, 1833) \\
\hline Sparassidae & Micrommata Latreille, 1804 & Micrommata virescens (Clerck, 1757) \\
\hline \multirow[t]{16}{*}{ Philodromidae } & Philodromus Walckenaer, 1826 & Philodromus aureolus (Clerck, 1757) \\
\hline & & Philodromus cespitum (Walckenaer, 1802) \\
\hline & & Philodromus collinus C. L. Koch, 1835 \\
\hline & & Philodromus dispar Walckenaer, 1826 \\
\hline & & Philodromus emarginatus (Schrank, 1803) \\
\hline & & Philodromus fallax Sundevall, 1833 \\
\hline & & Philodromus fuscomarginatus (De Geer, 1778) \\
\hline & & Philodromus histrio (Latreille, 1819) \\
\hline & & Philodromus margaritatus (Clerck, 1757) \\
\hline & & Philodromus poecilus (Thorell, 1872) \\
\hline & & Philodromus vagulus Simon, 1875 \\
\hline & Thanatus C. L. Koch, 1837 & Thanatus arenarius L. Koch, 1872 \\
\hline & & Thanatus formicinus (Clerck, 1757) \\
\hline & & Thanatus striatus C. L. Koch, 1845 \\
\hline & Tibellus Simon, 1875 & Tibellus maritimus (Menge, 1875) \\
\hline & & Tibellus oblongus (Walckenaer, 1802) \\
\hline \multirow[t]{12}{*}{ Thomisidae } & Coriarachne Thorell, 1870 & Coriarachne depressa (C. L. Koch, 1837) \\
\hline & Diaea Thorell, 1869 & Diaea dorsata (Fabricius, 1777) \\
\hline & Ebrechtella Dahl, 1907 & Ebrechtella tricuspidata (Fabricius, 1775) \\
\hline & Misumena Latreille, 1804 & Misumena vatia (Clerck, 1757) \\
\hline & Ozyptila Simon, 1864 & Ozyptila atomaria (Panzer, 1801) \\
\hline & & Ozyptila brevipes (Hah, 1826) \\
\hline & & Ozyptila gertschi Kurata, 1944 \\
\hline & & Ozyptila praticola (C. L. Koch, 1837) \\
\hline & & Ozyptila scabricula (Westring, 1851) \\
\hline & & Ozyptila trux (Blackwall, 1846) \\
\hline & Xysticus C. L. Koch, 1835 & Xysticus acerbus Thorell, 1872 \\
\hline & & Xysticus audax (Schrank, 1803) \\
\hline
\end{tabular}

continued 
Table 1. continued

\begin{tabular}{|c|c|c|}
\hline \multirow[t]{13}{*}{ Family } & Genus & Species \\
\hline & & Xysticus bifasciatus C. L. Koch, 1837 \\
\hline & & Xysticus chippewa Gertsch, 1953 \\
\hline & & Xysticus cristatus (Clerck, 1757) \\
\hline & & Xysticus erraticus (Blackwall, 1834) \\
\hline & & Xysticus kochi Thorell, 1872 \\
\hline & & Xysticus lanio C. L. Koch, 1835 \\
\hline & & Xysticus lineatus (Westring, 1851) \\
\hline & & Xysticus luctuosus (Blackwall, 1836) \\
\hline & & Xysticus obscurus Collett, 1877 \\
\hline & & Xysticus robustus (Hahn, 1832) \\
\hline & & Xysticus sabulosus (Hahn, 1832) \\
\hline & & Xysticus ulmi (Hahn, 1831) \\
\hline \multirow[t]{38}{*}{ Salticidae } & Aelurillus Simon, 1884 & Aelurillus v-insignitus (Clerck, 1757) \\
\hline & Dendryphantes C. L. Koch, 1837 & Dendryphantes hastatus (Clerck, 1757) \\
\hline & & Dendryphantes rudis (Sundevall, 1833) \\
\hline & Euophrys C. L. Koch, 1834 & Euophrys frontalis (Walckenaer, 1802 ) \\
\hline & & Euophrys herbigrada (Simon, 1871) \\
\hline & Evarcha Simon, 1902 & Evarcha arcuata (Clerck, 1757) \\
\hline & & Evarcha falcata (Clerck, 1757) \\
\hline & & Evarcha laetabunda (C. L. Koch, 1846) \\
\hline & Heliophanus C. L. Koch, 1833 & Heliophanus aeneus (Hahn, 1832) \\
\hline & & Heliophanus auratus C. L. Koch, 1835 \\
\hline & & Heliophanus cupreus (Walckenaer, 1802) \\
\hline & & Heliophanus dubius C. L. Koch, 1835 \\
\hline & & Heliophanus flavipes (Hahn, 1832) \\
\hline & & Heliophanus patagiatus Thorell, 1875 \\
\hline & Leptorchestes Thorell, 1870 & Leptorchestes berolinensis (C. L. Koch, 1846) \\
\hline & Marpissa C. L. Koch, 1846 & Marpissa muscosa (Clerck, 1757) \\
\hline & & Marpissa radiata (Grube, 1859$)$ \\
\hline & Myrmarachne MacLeay, 1839 & Myrmarachne formicaria (De Geer, 1778) \\
\hline & Neon Simon, 1876 & Neon levis (Simon, 1871) \\
\hline & & Neon reticulatus (Blackwall, 1853) \\
\hline & & Neon valentulus Falconer, 1912 \\
\hline & Pellenes Simon, 1876 & Pellenes tripunctatus (Walckenaer, 1802) \\
\hline & Phlegra Simon, 1876 & Phlegra fasciata (Hahn, 1826) \\
\hline & Pseudeuophrys Dahl, 1912 & Pseudeuophrys erratica (Walckenaer, 1826) \\
\hline & Salticus Latreille, 1804 & Salticus cingulatus (Panzer, 1797) \\
\hline & & Salticus scenicus (Clerck, 1757) \\
\hline & & Salticus zebraneus (C. L. Koch, 1837) \\
\hline & Sibianor Logunov 2001 & Sibianor aurocinctus (Ohlert, 1865) 1868 \\
\hline & Sitticus Simon, 1901 & Sitticus caricis (Westring, 1861) \\
\hline & & Sitticus distinguendus (Simon, 1868) (syn. Attulus helveolus) \\
\hline & & Sitticus floricola (C. L. Koch, 1837) \\
\hline & & Sitticus pubescens (Fabricius, 1775) \\
\hline & & Sitticus saltator (O. Pickard-Cambridge, 1868) \\
\hline & & Sitticus terebratus (Clerck, 1757) \\
\hline & Synageles Simon, 1876 & Synageles hilarulus (C. L. Koch, 1846) \\
\hline & & Synageles venator (Lucas, 1836$)$ \\
\hline & Talavera Peckham, Peckham 1909 & Talavera aequipes (O. Pickard-Cambridge, 1871) \\
\hline & & Talavera parvistyla Logunov, Kronestedt, 2003 \\
\hline
\end{tabular}


misera (O. P.-Cambridge, 1882), Centromerus levitarsis (Simon, 1884), Tapinocyba biscissa (O. P.-Cambridge, 1872), Taranucnus setosus (O. P.-Cambridge, 1863) (Linyphiidae) and Synema globusum (Fabricius, 1775), Thomisus onustus Walckenaer, 1805 (Thomisidae). Species Pardosa hyperborea (Thorell, 1872) (Lycosidae) is included in the list, but there is no reference on it from Latvia (registed in Fauna Europea, van Helsdingen 2017). .

Šternbergs collected spiders by use of a soil frame (or biocenometr) and hand sorting, while Cera, Spungis and Štokmane used pitfall traps and entomological sweep net for spider collecting. Some of the species were recently discovered from photo images (Dabasdati 2013; Rustanoviča 2014; Dabasdati 2015).

The spider taxonomy of this article follows the World Spider Catalogue 2018, version 19.0 (2018).

\section{Results and discussion}

This paper presents the checklist of all discovered spider species in the territory of Latvia. The checklist includes 500 spider species and 31 families (Table 1). Most probably the number of spider species in Latvia exceeds 500, as in Estonia there are 525 spider species (Meriste, pers. comments); fewer spider species are known for Lithuania - 445 (Biteniekyte, Relys 2011). Some species inhabiting non-studied layers of vegetation or living in other (not investigated) habitats can still remain as unknown for the Latvian fauna. In last two decades spiders were investigated more intensively thus resulting in more than 30 new spider species added to the list. These were found in investigations of peat bogs, calcareous fens and coastal habitats like dunes and meadows (Cera, Spungis 2008; 2009; 2010; Cera 2013; Štokmane et al. 2013 etc.).

\section{Acknowledgements}

This work was supported by European Social Fund (ESF) within the Project "New interdisciplinary group of scientists for resource exploration sustainable use and protection of Latvian mires" Nr. 2014/0009/1DP/1.1.1.2.0/13/APIA/VIAA/044 in the Institute of Biology, University of Latvia. The author is thankful to Oskars Keišs and Voldemārs Spuñgis (University of Latvia, Institute of Biology and Faculty of Biology) for help in manuscript correction and improvement.

\section{References}

Ancipanova J., Sternbergs M. 1984. Peculiarities of the development of the spider Philodromus aureolus (Cl., 1757) (Aranei, Thomisidae). Proc. Latv. Univ. Agricult. 213: 5-9. /in Russian/

Ancipanova J., Sternbergs M. 1987. Feeding of the dominant species of spiders (Aranei) in apple-tree agrocenosis. Proc. Latv. Univ. Agricult. 237: 10-14. /in Russian/

Biteniekytė M., Rèlys V. 2011. The checklist of Lithuanian spiders (Arachnida: Araneae). Biologija 57: 148-158.

Brūvele D. 1993. Soil spiders (Aranei) in the Teiči bog. University of Latvia, Riga, Bachelor thesis, 37 p. /in Latvian/
Cera I. 2008. First record of Hyptiotes paradoxus (C. L. Koch, 1834)

(Araneae: Uloboridae) in Latvia. Latvijas Entomologs 46: 79.

Cera I. 2009. Four spider (Araneae) species new to the fauna of Latvia. Latvijas Entomologs 47: 93-94.

Cera I. 2013. Fauna and distribution of spiders at Randu meadows, Baltic Sea coast of the Gulf of Riga, Latvia. Latvijas Entomologs 52: 68-83.

Cera I., Keišs O. 2016. Spider (Araneae) species new to the fauna of Latvia and new localities Gnaphosa lapponum and G. nigerrima (Gnaphosidae) in Natura 2000 sites. Latvijas Entomologs 53: 122-124.

Cera I., Spungíis V. 2008. Spider (Araneae) species new to the fauna of Latvia. Latvijas Entomologs 45: 49.

Cera I., Spungíis V. 2010. Distribution of spiders in dune habitats at the Baltic Sea coast at Akmensrags, Latvia. Latvijas Entomologs 49: 3-13.

Cera I., Spungigis V. 2013. Fauna and seasonal activity of spiders (Araneae) in coastal dune habitats at Akmensrags, Ziemupe Nature restricted territory, Latvia. Latvijas Entomologs 52: 84-95.

Cera I., Spungigis V., Melecis V. 2010. Occurrence of grass-dwelling spiders in different habitats at Lake Engure Nature Park. Environ. Exp. Biol. 8: 59-69.

Dabasdati 2013. Anelosimus pulchellus. Online at https:// dabasdati.lv/lv/observation/rgt018hv1mueqcoc3vbe6545m2/ /Accessed May 24, 2017/

Dabasdati 2015. New spider family and species found in Latvia. Online at http://www.dabasdati.lv/lv/article/atrasta-jaunazirneklu-dzimta-un-suga-latvija/. /Accessed June 15, 2015/

Fedorova N. 1991. Slīteres rezervāta vējgāzes zemsedzes zirnekḷi (Aranei). University of Latvia, Rìga, Bachelor thesis, 42 p. /in Russian/

Foelix R.F. 1996. Biology of Spiders. Georg Thime Verlag, New York. 330 p.

Golubeva O. 1997. Investiagtion of fauna and ecology of epigeic spider (Aranei) of pine forest near the Mazsalaca. University of Latvia, Riga, Master thesis, 53 p. /in Russian/

Grube A.E. 1859. Verzeichnis der Arachnoiden Liv-, Kur- und Estlands. Arch. Naturk. Liv-, Est- un Kurlands. Ser. 2, Bd 1, S. 414-486.

Karps A., Lapina M., Melecis V., Spungis V., Stenbergs M. 1990b. Environmental pollution by the waste water released from a pig-breeding complex. Digest of articles. Riga, pp. 81-83; 102-108. /in Russian/

Kušķe I. 1992. Epigeic spiders in decidious lime tree forest of nature reserve Moricsala. University of Latvia, Rìga, Bachelor thesis, 45 p. /in Latvian/

Kušners E. 1995. Fauna and flora of Latvian caves. University of Latvia, Rīga, Bachelor thesis, 73 p. /in Latvian/

Marpissa muscosa 2017. Collection of Latvian Museum of Natural History. Online at http://nmkk.lv/Items/ItemViewForm. aspx?id=258880. /Accessed July 25, 2015/

Mihnevic O. , Volkov D., Baumane L. 1990. Study of fauna and ecology of invertebrates in garden rotation experimental farm „Skrivveri”. In “To develop and introduce ways of effective use of useful organisms and products of their vital activity against pests, diseases and weeds", pp. 183-231. (manuscript, kept in Institute of Biology, Laboratory of Experimental Entomology)

Mihnevic O., Volkov D., Baumane L. 1989. Fauna of arthropods (Coleoptera, Hemiptera, aranei) in rapeseed field catched by pitfall traps. In „To develop and introduce methods of effective use of beneficial organisms and products of their vital activity 
against pests, diseases and weeds", pp. 107-136. /manuscript, kept in Institute of Biology, Laboratory of Experimental Entomology/

Narodickaja J. 1997. Investiagtion of fauna and ecology of grassliving spider (Aranei) of pine forest near the Mazsalaca. University of Latvia, Rìga, Master thesis, 53 p. /in Russian/

Neon levis 2015. Collection of Latvian Museum of Natural History. Online at http://nmkk.lv/Items/ItemViewForm. aspx?id=258903. /Accessed July 25, 2015/

Neon pictus 2015. Collection of Latvian Museum of Natural History. Online at http://nmkk.lv/Items/ItemViewForm. aspx?id=258922. /Accessed July 25, 2015/

Petrova V., Voitkane S., Jankevica L., Cera I. 2013. Spider community of the horse-chestnut Aesculus hippocastanum L. - preliminary results. Acta Biol. Univ. Daugavpil. 13: 77-84.

Petrovics N. 1995. Changes in the fauna of spiders (Aranei) in the Baži bog of the Slitere Reserve after the forest fire of 1992. University of Latvia, Rīga, Bachelor thesis, 29 p. /in Latvian/

Prieditis A., Sternbergs M. 1981. Spider fauna (Aranei) in appletree agrocenosis. Proc. Latv. Univ. Agricult. 188: 9-12. /in Russian/

Prieditis A., Sternbergs M. 1982. The lower level of activity of some spider species. Proc. Latv. Univ. Agricult. 200: 25-28. /in Russian/

Relys V., Spungisis V. 2002. Check list of spiders (Arachnida, Araneae) of Latvia. Online at http://leb.daba.lv/Aranea.htm. /Accessed June 12, 2015)/

Rustanoviča N. 2014. Velvet spider - new spider species for Latvia] Online at http://www.dabasdati.lv/lv/article/mariszirneklisndash-jauna-suga-latvijas-fauna/2014/. /Accessed July 24, 2015/

Samulaka B. 1996. Spiders (Araneae) of the oaten's and barley's agrobiocenozies in the vicinity of the Skriveri.] University of Latvia, Rìga, Bachelor thesis, 34 p. (in Russian)

Segestria senoculata 2015. Collection of Latvian Museum of Natural History. Online at http://nmkk.lv/Items/ItemViewForm. aspx?id=259311 (Accessed July 25, 2015)

Šestáková A., Krumpál M. 2013. First record of spider Gibbaranea omoeda (Thorell, 1870) (Araneae: Araneidae) to the fauna of Latvia. Latvijas Entomologs 52: 96-98.

Sevcenko I. 1991. Litter-dwelling spiders (Aranei) of ancient iceage Baltic lake shore in „Slitere"reserve. University of Latvia, Rìga, Bachelor thesis, 62 p. /in Russian/

Smalinskis J. 1994.Fauna and flora of Latvian caves. University of Latvia, Rìga, Bachelor thesis, 99 p. /in Latvian/

Spungis V.2008. Fauna and ecology of invertebrates (Invertebrata) in Slitere national park habitats. University of Latvia Academic Publishing, Rīga, 59 p. /in Latvian/

Spungigis V. 2005. Wasp spider Argiope bruennichi (Scopoli, 1772) (Aranea, Araneidae) in Latvia. Latvijas Entomologs 42: 106107.

Spungis V., Biteniekyte M., Relys V. 2005. The first year spider (Arachnida: Araneae) community in a burned area of Sudas bog in Latvia. Ekologija 1: 43-50.

Sternbergs M. 1974. Review of the spider fauna of Latvia. 1. Family Salticidae. Latvijas Entomologs 16: 65-70. /in Russian/

Sternbergs M. 1976. Materials on spider fauna of Latvia 2. Family Lycosidae. Latvijas Entomologs 18: 55-60. /in Russian/

Šternbergs M. 1977. Review of the spider fauna of Latvia. 3. Family Tetragnathidae. Latvijas Entomologs 20: 73-80. /in Latvian/

Sternbergs M. 1979a. Structure and dynamics of the spiderfauna of Aegopodio-Tilietum litter in "Moritssala" Reserve. In Fauna and Ecology of Arachnids. Works of Zoological Institute of the USSR Academy of Sciences 85: 54-59. /in Russian/

Šternbergs M. 1979b. Review of the spider fauna of Latvia. 4. The family Thomisidae. Latvijas Entomologs 22:73-77. /in Latvian/

Šternbergs M. 1980a. About local population finding of Oecobius annulipes Lucas, 1846 (Aranei, Oecobiidae) in Latvia. In: Latvian SSR Invertebrate Fauna and Ecology. P. Stuckas Univeristy of Latvia, Rīga, pp. 86-87. /in Latvian/

Šternbergs M. 1980b. Spiders in collection materials of ichneumonolog E. Ozols from vicinity of Priekul̦i. In: Latvian SSR Invertebrate Fauna and Ecology. P. Stuckas Univeristy of Latvia, Riga, pp. 88-90. /in Latvian/

Šternbergs M. 1980c. Epigeic spiders (Aranei) of black alder rich deciduous forest in nature rezerve "Moricsala”. Thesis of the $1^{\text {st }}$ practical and the scientific conference of the Slitere Reserve, pp. 23-24. /in Latvian/

Šternbergs M. 1981a. The spider in the litter of black alder rich deciduous forest in nature reserve "Moricsala". Forestry and Wood Industry 3: 40-42. /in Latvian/

Šternbergs M. 1981b. Review of the spider fauna of Latvia. 2. Family Clubionidae. Latvijas Entomologs 24: 56-59. /in Latvian/

Sternbergs M. 1982a. Spiders (Aranei) oft he litter of spruce forest communities in Tolkas Island, Kala Lake. In: Study of the Protected Nature Territories of the Latvian SSR. Zinatne, Riga, pp. 89-91./in Russian/

Sternbergs M. 1982b. Spiders (Aranei) of oak forest litter of "Moritssala" Reserve. In: Study of the Protected Nature Territories of the Latvian SSR. Zinatne, Riga, pp. 86-88. /in Russian/

Sternbergs M. 1983. Spiders (Chelicerata, Aranei. In: Nature Reserve Moricsala, Flora and Fauna. Riga, Avots, Riga, pp. 41-47./in Russian/

Sternbergs M. 1984. Structure and dynamics oflhe spider (Aranei) fauna of the litter of greenmoss spruce forest. Entomological Review 63: 188-192./in Russian/

Sternbergs M. 1985a. Effect of cement factory wastes on the spiders (Aranei) of the forest litter. In: Contamination of the Natural Environment with Calcium-containing Dust. Zinatne, Riga, pp. 101-109. /In Russian/

Šternbergs M. 1985b. Review of the spider fauna of Latvia. 6. Family Theridiidae. Latvijas Entomologs 28: 32-37. /in Latvian/

Šternbergs M. 1986. Review of the spider fauna of Latvia. 7. The family Linyphiidae. Latvijas Entomologs 29: 38-44. /in Latvian/

Šternbergs M. 1987. Grass-level spiders in open landscape biocenozis in Gauja ancient valley at Sigulda. Forestry and Wood Industry 3: 6-10. /in Latvian/

Šternbergs M. 1988. Review of the spider fauna of Latvia. 8. The family Micryphantidae. Latvijas Entomologs 31: 41-49. /in Latvian/

Sternbergs M. 1989. Effect of road transport pollution on the spiders (Aranei) of the natural grasslands. In: Impact of Motor Vehicle Vibration on the Environment. Zinatne, Riga, pp. 102107. /in Russian/

Šternbergs M. 1990a. Review of the spider fauna of Latvia. 9. The family Gnaphosidae. Latvijas Entomologs 33: 27-30. /in Latvian/

Šternbergs M. 1991. Epigeic spiders of Bažu bog at nature reserve Slìtere. Forestry and Wood Industry 1:37-43.

Šternbergs M. 1995a. The spiders (Aranei) in the litter of Fraxinetum dryopterioso forest type in the 'Slitere' Nature 
Reserve. In: Rủžička V. (ed) Proceedings of $15^{\text {th }}$ European Colloquium of Arachnology Institute of Entomology, Česke Budějovice, pp. 169-171.

Šternbergs M. 1995b. Trembling spider family (Pholcidae). In: Latvian Natural Encyclopedia, No. 2. Latvijas Enciklopedija, Riga, p. 93. /in Latvian/

Šternbergs M. 1995c. Giant crab spider family (Sparassidae). In: Latvian Natural Encyclopedia, No. 3. Latvijas Enciklopedija, Riga, p. 209. /in Latvian/

Šternbergs M. 1998. Materials on Latvian spider fauna. 10. Families Dictynidae, Agelenidae. Latvijas Entomologs 36: 27-30. /in Latvian/

Štokmane M. 2013. Ecological factor influence on epigeic and grass-dwelling spider communities in the Apšuciem's calcareous fen. University of Latvia, Riga, Master thesis, 56 p. /in Latvian/

Štokmane M., Spungigis V. 2014. Diversity of grass-dwelling spiders (Arachnida: Araneae) in calcareous fens of the Coastal Lowland, Latvia. J. Insect Conserv. 18: 757-769.

Štokmane M.,Spungóis V., Cera I.2013.Spider (Arachnida:Araneae) species richness, community structure and ecological factors influencing spider diversity in the calcareous fens of Latvia. Proceedings of the $54^{\text {th }}$ International Scientific Conference of Daugavpils University, pp. 45-55.

Šulmeistere D. 1998. Fauna and ecology of spiders of Lielupe river flood meadow near Kalnciems. University of Latvia, Rīga, Bachelor thesis, 48 p. /in Latvian/

Talavera aequipes 2015. Collection of Latvian Museum of Natural History. Online at http://nmkk.lv/Items/ItemViewForm. aspx?id=258922. $/$ Accessed July 15, 2015/

Tkaceva A. 1996. Spiders (Araneae) of the barley-clover's and winter rape's agrobiocenosies in the vicinity of the Skriveri. University of Latvia, Rìga, Bachelor thesis, 34 p. /in Russian/

van Helsdingen P.J. 2017. Araneae. In: Fauna Europaea Database European spiders and their distribution. Version 2017.1, date of publication February 10, 2017.

Vārtina I. 1992. Structure of fauna and dynamics of spiders in some agobiocenosis. University of Latvia, Riga, Bachelor thesis, 40 p. /in Latvian/

World Spider Catalog 2018. World spider catalog, version 19.0 Online at http://www.wsc.nmbe.ch/dataresources. /Accessed March 20, 2018/ 